\title{
Quantifying Double-Layer Potentials at Liquid-Gas Interfaces from Vibrational Sum-Frequency Generation
}

\author{
Natalia García Rey, ${ }^{\circledR}$ Eric Weißenborn, Felix Schulze-Zachau, Georgi Gochev, \\ and Björn Braunschweig*(i)
}

Institute of Physical Chemistry, Westfälische Wilhelms-Universität Münster, Corrensstraße 28/30, 48149 Münster, Germany

\section{Supporting Information}

ABSTRACT: Vibrational sum-frequency generation (SFG) spectroscopy is demonstrated as a fast method to quantify variations of the electric double-layer potential $\phi_{0}$ at liquid-gas interfaces. For this, mixed solutions of nonionic tetraethyleneglycol-monodecylether $\left(\mathrm{C}_{10} \mathrm{E}_{4}\right)$ and cationic hexadecyltrimethylammonium bromide $\left(\mathrm{C}_{16} \mathrm{TAB}\right)$ surfactants were investigated using SFG spectroscopy and a thin-film pressure balance (TFPB). Derjaguin-Landau-Verwey-Overbeek analysis of disjoining pressure isotherms obtained with the TFPB technique provides complementary information on $\phi_{0}$, which we apply to validate the results from SFG spectroscopy. By using a single $\phi_{0}$ value, we can disentangle $\chi^{(2)}$ and $\chi^{(3)}$ contributions to the $\mathrm{O}-\mathrm{H}$ stretching modes of interfacial water molecules in the SFG spectra. Having established the latter, we show that unknown double-layer potentials at the liquid-gas interface from solutions with different $\mathrm{C}_{16} \mathrm{TAB} / \mathrm{C}_{10} \mathrm{E}_{4}$ mixing ratios can be obtained from an analysis of SFG spectra and are in excellent agreement with the complementary results from the TFPB technique.

\section{INTRODUCTION}

The interaction of ions, surfactants, or proteins can significantly change the surface chemistry of aqueous interfaces and is of great importance for a wide range of fundamental questions in the physical chemistry of interfaces as well as in applications. ${ }^{1-4}$ In fact, there is a general interest to quantify the charging state of fluid interfaces in applications ranging from antibody formulation to oxide surfaces. ${ }^{5-8}$ Particularly, in soft colloids, such as foams and emulsions, the electrostatic component can dominate the disjoining pressure $\Pi, 9,10$ which stabilizes bubbles or drops against coalescence. ${ }^{9-12}$ Addressing as well as quantifying double-layer potentials $\phi_{0}$ at charged interfaces is, therefore, a necessity.

However, for investigations of aqueous interfaces, there exist only a few experimental techniques that can probe their charging state and provide quantitative information on $\phi_{0}$. For solid/liquid interfaces, classical methods such as the surfaceforce apparatus ${ }^{13}$ and (colloidal probe) atomic force microscopy ${ }^{14-16}$ have been used to provide information on $\phi_{0}$ by an analysis of force-distance curves and the application of the Derjaguin-Landau-Verwey-Overbeek (DLVO) theory. For liquid/gas interfaces, the use of the thin-film pressure balance (TFPB) technique ${ }^{17,18}$ is so far the only commonly accepted method for quantitative analysis but is also constrained to stable foam or emulsion thin films. In the present work, we address the possibilities to quantify $\phi_{0}$ at charged water interfaces using sum-frequency generation (SFG) and directly compare the results with a complementary method, which has lately attracted considerable interest ${ }^{19-23}$ and traces back to the original work by Eisenthal and coworkers $^{24-27}$ using second-harmonic generation (SHG). In addition to SHG, the applicability of SFG for charge determination at interfaces was demonstrated. ${ }^{7,21,28-31}$ However, validation on the limits and boundaries of SFG using a complementary technique such as the TFPB has hitherto not been presented but will be addressed in this work using ionic surfactant and nonionic surfactant mixtures to systematically change $\phi_{0}$ at the liquid-gas interface (Figure 1). It is wellknown that surfactants provide colloidal stability and stability of foams. ${ }^{32}$ Particularly, mixtures of surfactants are used to modify the properties of interfaces, ${ }^{33,34}$ foam films, ${ }^{35}$ and thereby the properties of macroscopic foam. ${ }^{34,36}$

For example, addition of a (less soluble) co-surfactant to a surfactant solution provides increased interfacial dilational viscoelasticity. ${ }^{32,33}$ With respect to charge-induced interfacial properties, mixed solutions of nonionic and ionic surfactants in different molar ratios can be used as a tool to obtain stable foam films, while simultaneously the charge density at the film interfaces and thus the electrostatic interactions in the films can be controlled in a well-defined way. ${ }^{35}$

On the basis of the double-layer theory of a charged airwater interface, ${ }^{1}$ the interfacial region is composed of a few monolayers of molecules, followed by bulk like water in the socalled diffuse layer. However, neither the electric double-layer

Received: October 16, 2018

Revised: December 13, 2018

Published: December 20, 2018 


\section{(a)}
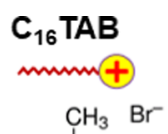

$\mathrm{H}_{3} \mathrm{C}\left(\mathrm{H}_{2} \mathrm{C}\right)_{15}-\stackrel{\mathrm{N}^{+}-\mathrm{CH}_{3}}{\mathrm{C}}$
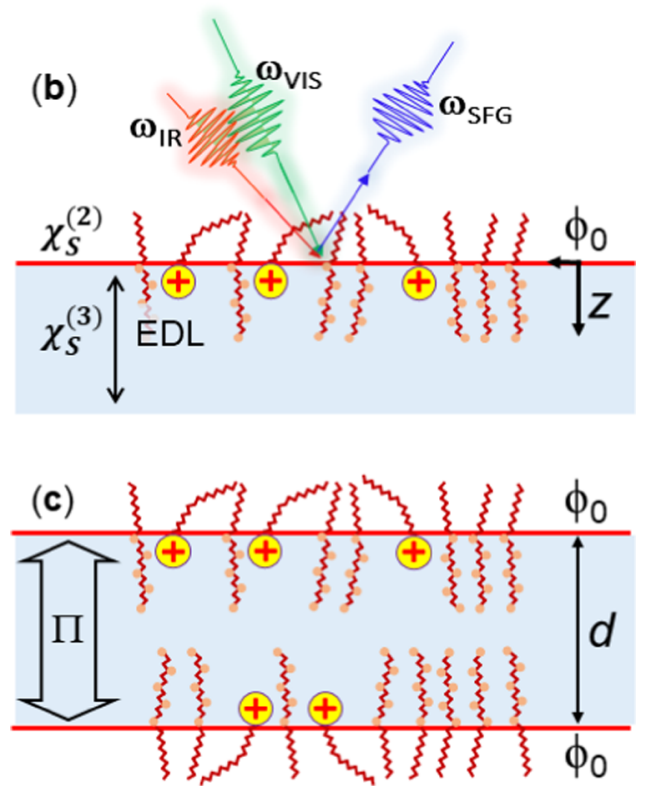

Figure 1. (a) Chemical structures and artistic sketches of $\mathrm{C}_{16} \mathrm{TAB}$ and $\mathrm{C}_{10} \mathrm{E}_{4}$ surfactants used in this study. Artistic sketch (not to scale) of (b) an air-charged liquid interface and (c) a foam film from mixed surfactant solutions showing key parameters of the two techniques employed in this manuscript: (b) sum-frequency generation spectroscopy and (c) thin-film pressure balance.

potential $\phi_{0}$ nor the corresponding surface charge of the liquid-gas interface are directly accessible by available methods and without formulation of a consistent molecularlevel description of the interface. In this respect, much progress in molecular-level understanding of aqueous interfaces has been developed due to the fruitful feedback between experiments and simulations. ${ }^{31,37-44}$

Although previous studies already used a combination of SFG and TFPB, ${ }^{12,22}$ the authors applied SFG mainly to address the structure of the interface but not to extract quantitative information on $\phi_{0}$. In fact, the relation between the SFG intensity of $\mathrm{O}-\mathrm{H}$ stretching bands and $\phi_{0}$ has not been discussed quantitatively in these works. In a recent work by Dreier et al., ${ }^{23}$ SHG spectroscopy was combined with the vibrating plate capacitor method on a lipid monolayer to extract $\phi_{0}$. However, the authors concluded that the two approaches were dominated by different molecular moieties and effects. Including SFG in their analysis, Dreier et al. ${ }^{23}$ showed how the SHG signals were dominated by contributions of interfacial water molecules, the lipids, as well as by hyperRayleigh scattering, whereas the response from the vibrating plate capacitor was attributed directly to the lipid carbonyl groups. In fact, Dreier et al. $^{23}$ pointed out that the interpretation of $\phi_{0}$ from SHG spectroscopy and its comparison with other methods need to be carefully analyzed and that a comparison at least between the latter two methods is highly challenging.

We have addressed air-water interfaces from mixed solutions that contained the nonionic surfactant tetraethyle- neglycol-monodecylether $\left(\mathrm{C}_{10} \mathrm{E}_{4}\right)$ and the cationic surfactant hexadecyltrimethylamonium bromide $\left(\mathrm{C}_{16} \mathrm{TAB}\right)$ (Figure $\left.1 \mathrm{a}\right)$. In this system, $\mathrm{C}_{16} \mathrm{TAB}$ is the charge-determining species at the interface and its surface excess thus controls $\phi_{0}$. Thermodynamic models describe the adsorption behavior of mixed surfactant systems; however, separating the surface excess from the nonionic and ionic species is not that simple. ${ }^{4,46}$ Both surfactants were chosen because of their known surface properties $^{47-50}$ and their close critical micelle concentrations, which are $\sim 0.9^{47}$ and $\sim 0.7 \mathrm{mM}^{48}$ for $\mathrm{C}_{16} \mathrm{TAB}$ and $\mathrm{C}_{10} \mathrm{E}_{4}$, respectively. Our strategy demonstrates how the change in the $\mathrm{O}-\mathrm{H}$ amplitude $\left(A_{\mathrm{OH}}\right)$ from interfacial $\mathrm{H}_{2} \mathrm{O}$ can be used to determine $\phi_{0}$ quantitatively, whereas these results are corroborated with the results obtained from the TFPB technique (Figure 4).

\section{THEORY}

Vibrational SFG spectroscopy is a three-photon process where an infrared (IR) and a visible (VIS) beam are overlapped in time and space at the interface, generating the sum-frequency beam $\left(\omega_{\mathrm{SF}}=\omega_{\mathrm{IR}}+\omega_{\mathrm{VIS}}\right)$. The electric field of the induced second-order polarization at the sum frequency (SF) is proportional to the effective second-order polarization of the interface: $P^{(2)}\left(\omega_{\mathrm{SF}}\right) \propto \chi_{\mathrm{eff}}^{(2)} E_{\mathrm{IR}} E_{\mathrm{VIS}}$. At the air-water interface, the SF signal arises from both surface and bulk contributions (Figure 1b). ${ }^{51}$ The former contribution depends on the second-order electric susceptibility $\chi_{\mathrm{S}}^{(2)}$, which is dominated by the molecular structure of interfacial molecules. ${ }^{20,52-54}$ The latter is caused by the static electric field $E_{\mathrm{DC}}$ in the electric double layer (EDL) perpendicular to the interface $(z)$ and originates from adsorbed molecules that carry a net charge (surfactants, ${ }^{55}$ proteins, ${ }^{12}$ polyelectrolytes, etc.) and depends on the third-order electric susceptibility $\chi_{\mathrm{S}}^{(3)}$. In the EDL, reorientation and polarization of interfacial water molecules are possible. ${ }^{26,28,56-59}$ The effective second-order electric susceptibility $\chi_{\text {eff }}^{(2)}$ of the charged air-water interface can be expressed by $^{20}$

$$
\begin{aligned}
\chi_{\text {eff }}^{(2)}= & \chi_{\mathrm{S}}^{(2)}+\chi_{\mathrm{S}, \mathrm{EDL}}^{(2)} \\
\chi_{\mathrm{S}, \mathrm{EDL}}^{(2)} & =\int_{0}^{+\infty} \chi_{\mathrm{S}}^{(3)} E_{\mathrm{DC}}\left(z^{\prime}\right) \mathrm{e}^{-\mathrm{i} \Delta k_{z^{\prime}}} \mathrm{d} z^{\prime} \\
& =\chi_{\mathrm{S}}^{(3)} \int_{0}^{+\infty}\left(-\frac{\mathrm{d} \phi\left(z^{\prime}\right)}{\mathrm{d} z^{\prime}}\right) \mathrm{e}^{-\mathrm{i} \Delta k_{z^{\prime}}} \mathrm{d} z^{\prime}
\end{aligned}
$$

here, the $\chi_{\mathrm{S}, \mathrm{EDL}}^{(2)}$ term accounts for the probing depth and the phase mismatch $\Delta k_{z}$ of the fundamental and SF waves at charged interfaces $\left(E_{\mathrm{DC}} \neq 0\right)$, which reflects possible interference effects at low ionic strengths. ${ }^{20,21,57}$ Using the Gouy-Chapman model and the Debye-Hückel approximation to solve the Poisson-Boltzmann equation, we can write the double-layer potential $\phi(z)=\phi_{0} \mathrm{e}^{-\kappa z}$. Using the latter equation, one can obtain the relation between the $\chi_{\text {eff }}^{(2)}$ and $\phi_{0}$ by integration of eq $2^{7,20,21,30,56,60}$

$$
\begin{aligned}
I\left(\omega_{\mathrm{SF}}\right) & \left.\propto I\left(\chi_{\mathrm{eff}}^{(2)}\right)\right|^{2} I_{\mathrm{IR}} I_{\mathrm{VIS}} \\
& =\left|\left(\chi_{\mathrm{S}}^{(2)}+\frac{\kappa}{\kappa+\mathrm{i} \Delta k_{z}} \chi_{\mathrm{S}}^{(3)} \phi_{0}\right)\right|^{2} I_{\mathrm{IR}} I_{\mathrm{VIS}}
\end{aligned}
$$

To use the SF intensity $I\left(\omega_{\mathrm{SF}}\right)$ for quantification of $\phi_{0}$, direct application of eq 3 is, however, not possible because of the three unknown parameters: $\chi_{\mathrm{S}}^{(2)}, \chi_{\mathrm{S}}^{(3)}$, and $\phi_{0}$. Obviously, 
disentangling these parameters is necessary and we have, therefore, varied $\phi_{0}$ systematically, studied the changes in SF signals, and combined our analysis with thin-film pressure balance measurements.

\section{EXPERIMENTAL SECTION}

Sample Preparation. $\mathrm{C}_{16} \mathrm{TAB}$ (BioUltra, Sigma-Aldrich, $>99 \%$ ) and $\mathrm{C}_{10} \mathrm{E}_{4}$ (Bachem, Switzerland, 98.8\%) were used as received. Mixed surfactant solutions were prepared with a total concentration $C_{\text {surf }}=0.7 \mathrm{mM}$ but different $\left[\mathrm{C}_{16} \mathrm{TAB}\right] /\left[\mathrm{C}_{10} \mathrm{E}_{4}\right]$ molar ratios. The ionic strength of the solutions $I=0.7$ or 500 $\mathrm{mM}$ was adjusted with $\mathrm{NaCl}$ (BioXtra, Sigma-Aldrich, 99.9\%). Further details about the samples' composition and preparation are given in the Supporting Information.

Broadband Sum-Frequency Generation Spectrometer. Münster Ultra-fast Spectrometer for Interfacial Chemistry (MUSIC) is a user-friendly broadband sum-frequency spectrometer. MUSIC is composed of a Spectra Physics Solstice-Ace Ti:sapphire amplifier seeded by a Ti:sapphire fsoscillator and pumped by a Q-switched laser (Ascend 60, 520 $\mathrm{nm}, 1 \mathrm{kHz}, 32 \mathrm{~W})$. The oscillator emits at a wavelength of 794 $\mathrm{nm}$ and has a bandwidth of $22 \mathrm{~nm}$ (Spectra Physics MaiTai SP, $84 \mathrm{MHz}, 770 \mathrm{~mW}$ ). The Solstice-Ace regenerative amplifier delivers approximately $7 \mathrm{~W}(1 \mathrm{kHz}$, at $796 \mathrm{~nm})$ of average power. The uncompressed beam is divided by an internal (50:50) beam splitter. One beam is subsequently guided into an internal, and the second beam to an external compressor. A total power of $3.2 \mathrm{~W}$ of the amplified and compressed femtosecond beam (796, $18 \mathrm{~nm}$ bandwidth) pumps an optical parametric amplifier (OPA, Light conversion TOPAS Prime) with a subsequent unit for noncollinear difference frequency generation (NDFG) of the OPAs' idler and signal photons. The NDFG unit generates broadband femtosecond IR pulses that are tuneable from 2.5 to $20 \mu \mathrm{m}$. The broadband IR has $>300 \mathrm{~cm}^{-1}$ full width at half-maximum bandwidth. An airspaced etalon (SLS OPTics LTD, FSR $12.4 \mathrm{~nm}$ at $735 \mathrm{~nm}, R=$ $94.5 \%)$ was inserted into the external compressor to generate the VIS narrowband pulse centered at $804.1 \mathrm{~nm}$ and with a bandwidth of $4 \mathrm{~cm}^{-1}$. Etalon side bands are removed by beam blocks, which are also placed inside the external compressor.

For the SFG, the VIS and IR pulses overlap in time and space at 55 and $60^{\circ}$ incident angles, respectively. The mean circularized focused beam diameters $\left(1 / e^{2}\right)$ were 530 and 260 $\mu \mathrm{m}$ for the VIS and IR beams, using a VIS lens $f^{\prime}=+500 \mathrm{~mm}$ (N-BK7, Thorlabs LA1908-780) and an IR lens $f^{\prime}=+100 \mathrm{~mm}$ (ZnSe, AMSTechnologies ZC-PX-25-100 BB-AR coated).

The SFG photons were collected by a collimator lens $f^{\prime}=$ $+200 \mathrm{~mm}$ (N-BK7, Thorlabs LA1979-A) and focusing lens $f^{\prime}=$ $+100 \mathrm{~mm}$ (LA1509-A), directing the SFG beam into a spectrograph (Kymera-328i-D2-SIL, Andor) using a $1200 \mathrm{~g} /$ $\mathrm{mm}$ grating imaged into an electron-multiplied charge-couple device EMCCD (Andor Newton, Du97P-BVF). A short pass filter with the cutoff at $763 \mathrm{~nm}$ (AHF, F76-789) was used to filter the visible beam.

For the liquid-gas interface, all spectra were recorded using s-polarized SF, s-polarized visible, and p-polarized IR beams, that is, in the ssp polarization configuration. The polarization optics used are a half-wave plate for the VIS (Bernhard Halle Nachfl RZQ 2.15.0795) and a combination of an achromatic half-wave plate (Bernhard Halle Nachfl RAC 4.2.15) with a Glan polarizing prism of calcite (Bernhard Halle Nachfl, PGL 12). The Glan polarizing prism matches the polarization of the spectrograph-grating, being sensitivity-independent of the selected SFG polarization during the experiment.

The IR beam path and the sample-automatized stage are enclosed in a compressed air-purged box. The relative humidity inside the box is $<3 \%$.

The SFG spectra were taken scanning five IR frequencies from 2700 to $3700 \mathrm{~cm}^{-1}$. The spectra were stitched together and normalized with the SFG signal of an air-plasma-cleaned $\mathrm{Au}$ film that served as a reference. The SFG air-liquid spectra were performed with a glass Petri dish, containing $2.5 \mathrm{~mL}$ of the liquid. The glass was previously soaked in a mixed solution of sulfuric acid with Nochromix overnight, rinsed extensively with Milli-Q water, and dried with $\mathrm{N}_{2}$ afterward.

Thin-Film-Pressure-Balance (TFPB) Technique. To obtain disjoining pressure isotherms $\Pi(d)$, we utilize the porous plate technique, developed by Mysels ${ }^{61}$ and refined by Exerowa. ${ }^{18}$ The experimental setup is schematically shown in Figure S6. In our experiments, a foam film is created in a $2 \mathrm{~mm}$ hole of a porous glass plate, which is fused to a glass capillary tube ( $1 \mathrm{~mm}$ inner diameter). The film holder is enclosed in a gastight pressure cell with two $\mathrm{CaF}_{2}$ windows. The chamber is equipped with a reservoir filled up with the sample solution, avoiding evaporation of water from the foam film. The pressure is controlled by a syringe pump (Legato210, kdScientific) and measured by a pressure transducer (A-10, 0-50 mbar, WIKA Germany).

The disjoining pressure $\Pi$ in the film is calculated as follows

$$
\Pi(d)=P_{\mathrm{g}}-P_{\mathrm{r}}+2 \frac{\gamma}{r}-\Delta \rho g h_{\mathrm{c}}
$$

where $P_{\mathrm{g}}$ and $P_{\mathrm{r}}$ are the chamber and external pressures, respectively; $\gamma$, the surface tension; $r$, the radius of the glass tube's channel; $\Delta \rho$, the density difference between surfactant solution and air; $g=9.81 \mathrm{~m} / \mathrm{s}^{2}$; and $h_{\mathcal{c}}$, the height of solution in the glass tube above the film (see Figure S6). The third and fourth terms in the equation stand for the capillary pressure and the hydrostatic pressure in the capillary, respectively.

For a visual access of the foam film, a camera is mounted at the top of the microscope. Between the objective and the pressure chamber, a beam splitter is used to couple the focused broadband measurement beam from a deuterium-halogen lamp (AvaLight-D-S-Bal, Avantes). The transmission spectra $T(\lambda)$ allow to calculate the thickness of the film, as described in the Supporting Information, using a fiber-optical spectrometer (AVASPEC-ULS3648-USB2-UA-25, Avantes).

Results and Discussion. In our procedure, we have recorded SFG spectra for different $\left[\mathrm{C}_{16} \mathrm{TAB}\right] /\left[\mathrm{C}_{10} \mathrm{E}_{4}\right]$ mixtures (Figure 2) and we compare the spectra of the mixtures at a constant total surfactant concentration $\left(\left[\mathrm{C}_{16} \mathrm{TAB}\right]+\left[\mathrm{C}_{10} \mathrm{E}_{4}\right]\right)$ of $0.7 \mathrm{mM}$ and constant total ionic strengths $\left(\left[\mathrm{C}_{16} \mathrm{TAB}\right]+\left[\mathrm{C}_{10} \mathrm{E}_{4}\right]+[\mathrm{NaCl}]\right)$. As ionic strengths we have chosen low $0.7 \mathrm{mM}$ and high $500 \mathrm{mM}$ values for each surfactant mixing ratio. To clarify the composition of our samples, we refer to Table $S 1$ in the Supporting Information.

To analyze the changes of $\mathrm{O}-\mathrm{H}$ bands, we fit our spectra in Figure 2, accounting for the nonresonant $\chi_{\mathrm{NR}}^{(2)}$ and the resonant contributions, e.g., due to $\mathrm{C}-\mathrm{H}$ and $\mathrm{O}-\mathrm{H}$ vibrational modes. We use a combination of Lorentzian functions for the $\mathrm{C}-\mathrm{H}$ stretching modes $\left(2750-3000 \mathrm{~cm}^{-1}\right)$ and a Voigt function for the $\mathrm{O}-\mathrm{H}$ bands $\left(2950-3700 \mathrm{~cm}^{-1}\right)$, which accounts for both homogeneous and inhomogeneous line broadening. ${ }^{62}$ See the Supporting Information for further description and the fitting parameters. 


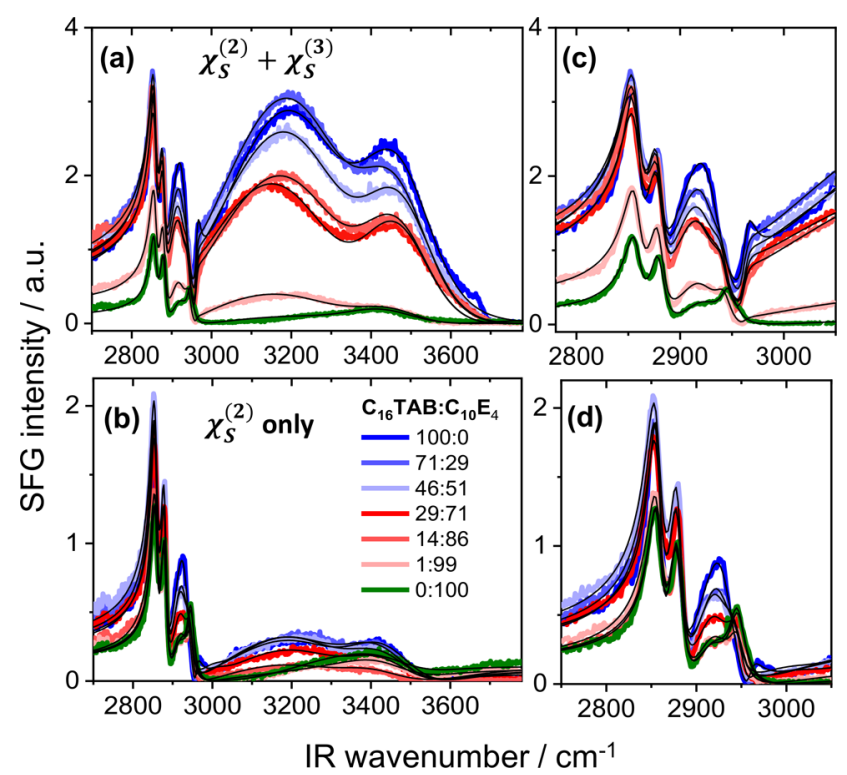

Figure 2. SFG spectra for different $\left[\mathrm{C}_{16} \mathrm{TAB}\right] /\left[\mathrm{C}_{10} \mathrm{E}_{4}\right] /[\mathrm{NaCl}]$ mixing ratios with a constant total surfactant concentration of $C_{\text {surf }}$ $=0.7 \mathrm{mM}$. The mole percentage of the mixtures was as indicated in the figure. Ionic strength was fixed by adding $\mathrm{NaCl}$ to (a) $I=0.7 \mathrm{mM}$ and to (b) $I=500 \mathrm{mM}$. (c) and (d) show a close-up of the $\mathrm{C}-\mathrm{H}$ bands in (a) and (b) respectively. All spectra were recorded using spolarized SF, s-polarized VIS, and p-polarized IR beams that are in the ssp polarization configuration.

The amplitudes from the $\mathrm{O}-\mathrm{H}$ stretching bands dominate the spectra in Figure 2a and appear as two separate broad bands centered at 3250 and $3400 \mathrm{~cm}^{-1}$. These bands have been previously attributed to $\mathrm{O}-\mathrm{H}$ stretching modes of tetrahedrally coordinated (low-frequency branch) and nontetrahedrally coordinated (high-frequency branch) interfacial $\mathrm{H}_{2} \mathrm{O}$ molecules. ${ }^{29,54,63}$ Tahara and co-workers ${ }^{28,64}$ concluded from phase-resolved SFG spectroscopy at cetyltrimethylammonium bromide/water interfaces without additions of $\mathrm{C}_{10} \mathrm{E}_{4}$ that the $\mathrm{O}-\mathrm{H}$ bands have negative amplitudes. The latter is direct proof that the interfacial water molecules exhibit a net orientation with the water's hydrogens pointing away from the interface toward the bulk solution (H-down orientation). Figure 2a clearly demonstrates that not only does the intensity of $\mathrm{O}-\mathrm{H}$ bands decrease as the $\left[\mathrm{C}_{16} \mathrm{TAB}\right] /\left[\mathrm{C}_{10} \mathrm{E}_{4}\right]$ molar ratio is decreased but also a change in the shape of the $\mathrm{O}-\mathrm{H}$ bands can be noticed from a close inspection of Figure $2 a, b$. This change in the $\mathrm{O}-\mathrm{H}$ spectrum is likely associated to a change in the water's surface chemistry due to changes in the surface excess of $\mathrm{C}_{16} \mathrm{TAB}$ relative to $\mathrm{C}_{10} \mathrm{E}_{4}$. The latter change in the surface excess can be seen most clearly by an inspection of the surfactants' $\mathrm{C}-\mathrm{H}$ bands, which we show in more detail in Figure $2 \mathrm{c}$,d. We observed methyl as well as methylene stretching bands centered at $\sim 2880$ and $\sim 2850 \mathrm{~cm}^{-1}$, respectively. In particular, the presence of strong methylene bands indicates a substantial number density of gauche conformations at the interface and that not all molecules are in an all-trans state. The latter would give rise to local inversion symmetry and thus negligible $\mathrm{CH}_{2}$ contributions to the SFG spectra. $^{64,65}$

We can now link the $\mathrm{O}-\mathrm{H}$ amplitude $\left|A_{\mathrm{OH}, \text { low }}\right|$ at $I=0.7 \mathrm{mM}$ to the effective interfacial susceptibility $\chi_{\text {eff }}^{(2)}$ for different mixture ratios (Figure $2 \mathrm{a}$ ). We recall that the latter is dependent on the double-layer potential $\phi_{0}$, as discussed above (eq 3).

$$
\begin{aligned}
& \left|\chi_{\text {eff }}^{(2)}\right|^{2} \propto\left|A_{\mathrm{OH}, \mathrm{low}}\right|^{2} \\
& \left|\chi_{\mathrm{eff}}^{(2)}\right|^{2}=\left|\chi_{\mathrm{S}}^{(2)}+\left(f_{1}-\mathrm{i} f_{2}\right) \chi_{\mathrm{S}}^{(3)} \phi_{0}\right|^{2}
\end{aligned}
$$

In eq 6 , factors $f_{1}$ and $f_{2}$ change with the Debye length $\kappa^{-1}$ but have negligible variation for the range of IR wavelengths used in our experiments (Figure S3). ${ }^{7,21}$ However, at high ionic strength $(I>200 \mathrm{mM})$, a substantial screening of the EDL takes place and decreases $\chi_{\mathrm{S}}^{(3)}$ contributions to negligible values $\left(\kappa \gg \Delta k_{z}, f_{1} \rightarrow 1\right.$ and $\left.f_{2} \rightarrow 0\right) .{ }^{27}$ This is consistent with recent works $^{7,66}$ and consequently $\chi_{\text {eff }}^{(2)}$ depends only on $\chi_{\mathrm{S}}^{(2)}$ contributions at high ionic strength. Having said this, we can now determine the first unknown parameter $\chi_{S}^{(2)}$ directly from our fitting results to the SFG spectra at high ionic strength.

$$
\left|A_{\mathrm{OH}, \mathrm{high}}\right|^{2} \sim\left|\chi_{\mathrm{S}}^{(2)}\right|^{2}
$$

In the second step, we can eliminate the second free parameter by introducing (for a single mixing ratio) a known double-layer potential $\left(\phi_{0, \mathrm{TFPB}}\right)$. This allows us to calculate $\left|\chi_{S}^{(3)}\right|$ from the previously determined $\left|\chi_{\mathrm{S}}^{(2)}\right|$ and $\left|A_{\mathrm{OH}, \text { low }}\right|$, which we retrieve from our fits to the SFG spectra at low and high ionic strengths (Figure 2a). However, a prerequisite for this analysis is that we need to include a phase $\delta$ between $\chi_{\mathrm{S}}^{(2)}$ and $\chi_{\mathrm{S}}^{(3)}$. At the charged water-air interface, the average molecular dipole orientation is perpendicular to the interface (the direction depends on positive or negative surface charge). Since a theoretical study of $\chi_{\mathrm{S}}^{(3)}$ of the air-water interface ${ }^{49}$ shows how the phase between $\chi_{S}^{(2)}$ and $\chi_{S}^{(3)}$ can have opposite signs, we consider both cases: 0 and $\pi$. After expansion of eq 3 that is presented in detail in the Supporting Information, the following expression can be derived

$$
\begin{aligned}
\left|\chi_{\text {eff }}^{(2)}\right|^{2}= & \left|\chi_{\mathrm{S}}^{(2)}\right|^{2}+2\left(f_{1} \cos (\delta)-f_{2} \sin (\delta)\right)\left|\chi_{\mathrm{S}}^{(2)} \| \chi_{\mathrm{S}}^{(3)}\right| \phi_{0} \\
& +\left(f_{1}^{2}+f_{2}^{2}\right)\left|\chi_{\mathrm{S}}^{(3)}\right|^{2} \phi_{0}^{2}
\end{aligned}
$$

From our fits to the SFG spectra in Figure 2, we determine I $A_{\mathrm{OH} \text {,high }} \mid$ and $\left|A_{\mathrm{OH}, \text { low }}\right|$. Inserting eqs 5 and 7 in 8 , one can simplify eq 8 to

$$
\begin{gathered}
\left|\chi_{\mathrm{S}}^{(3)}\right|^{2}+\frac{2\left(f_{1} \cos (\delta)-f_{2} \sin (\delta)\right)}{f_{1} \phi_{0, \mathrm{TFPB}}}\left|A_{\mathrm{OH}, \mathrm{high}}\right|\left|\chi_{\mathrm{S}}^{(3)}\right| \\
+\frac{\left|A_{\mathrm{OH}, \mathrm{high}}\right|^{2}-\left|A_{\mathrm{OH}, \mathrm{low}}\right|^{2}}{f_{1} \phi_{0, \mathrm{TFPB}}^{2}}=0
\end{gathered}
$$

where $\phi_{0, \text { TFPB }}$ from the TFPB is used for "calibration" or determination of the second unknown $\left|\chi_{S}^{(3)}\right|$. Considering the two possible scenarios where the phase $\delta$ between the susceptibilities $\chi_{\mathrm{S}}^{(2)}$ and $\chi_{\mathrm{S}}^{(3)}$ is either 0 or $\pi$, eq 9 can be rewritten as follows

$$
\begin{aligned}
\left|\chi_{\mathrm{S}}^{(3)}\right|= & \mp \frac{\left|A_{\mathrm{OH}, \text { high }}\right|}{\phi_{0, \mathrm{TFPB}}} \\
& +\sqrt{\frac{\left|A_{\mathrm{OH}, \mathrm{high}}\right|^{2}}{\left(\phi_{0, \mathrm{TFPB}}\right)^{2}}-\frac{\left|A_{\mathrm{OH}, \mathrm{high}}\right|^{2}-\left|A_{\mathrm{OH}, \mathrm{low}}\right|^{2}}{\left(\phi_{0, \mathrm{TFPB}}\right)^{2} f_{1}}}
\end{aligned}
$$

Obviously, for the different phases, we will get according to eq 10 also a different value for $\left|\chi_{\mathrm{S}}^{(3)}\right|$. Having established the $\chi_{\mathrm{S}}^{(3)}$ for one system (here, one mixing ratio of $\mathrm{C}_{16} \mathrm{TAB} / \mathrm{C}_{10} \mathrm{E}_{4}$ ), we 
can determine the double-layer potentials for unknown mixing ratios from an analysis of SFG spectra, using the following expression, derived from eq 8

$$
\begin{aligned}
\phi_{0, n}= & \mp \frac{\left|A_{\mathrm{OH}, \text { high }}\right|}{\left|\chi_{\mathrm{S}}^{(3)}\right|} \\
& +\sqrt{\frac{\left|A_{\mathrm{OH}, \mathrm{high}}\right|^{2}}{\left|\chi_{\mathrm{S}}^{(3)}\right|^{2}}-\frac{\left|A_{\mathrm{OH}, \mathrm{high}}\right|^{2}-\left|A_{\mathrm{OH}, \text { low }}\right|^{2}}{\left|\chi_{\mathrm{S}}^{(3)}\right|^{2} f_{1}}}
\end{aligned}
$$

To determine the double-layer potential as discussed above, we have to consider the following points: (i) $\chi_{\mathrm{S}}^{(2)}$ necessarily depends on the surfactant mixing ratio, which accounts for the structural changes of the interface. (ii) We assume $\chi_{S}^{(2)}$ has the same spectra at low and high ionic strengths for a given mixing ratio. This is also corroborated by the study of Urashima et al. $^{66}$ (iii) $\chi_{\mathrm{S}}^{(3)}$ spectra are independent of the surfactant mixing ratio, as was also pointed out earlier by Wen et al. ${ }^{20}$

We now discuss the point (ii) from the viewpoint of soft interfaces that are composed of different surfactants, which can change their composition with ionic strength. For nonionic surfactants, variations in the ionic strength do not lead to changes in the adsorption behavior. In contrast, the surface activity of ionic surfactants increases with ionic strength as the electrostatic repulsion between the charged surfactants is reduced. This leads to an increase of the surfactants' surface excess and to a decrease of the surfactants' critical micelle concentration. ${ }^{67-69}$ However, studies of ionic/nonionic surfactant mixtures have shown that for [ionic] $/[$ nonionic] molar mixing ratios $<1$, like in our work, the surface properties are dominated by the nonionic species. ${ }^{35,70}$ Moreover, we measured the surface tensions $\gamma$ of $\mathrm{C}_{16} \mathrm{TAB} / \mathrm{C}_{10} \mathrm{E}_{4}$ mixtures and found that $\gamma$ varies only within a few $\mathrm{mN} / \mathrm{m}$ if the mixing ratio is constant but the ionic strength is varied (see Figure S1). Therefore, surface tension indicates that variations in the total surface excess are very small as a function of ionic strength. Consequently, these results from surface tensiometry corroborate our earlier assumption that $\chi_{S}^{(2)}$ has a similar spectrum at different ionic strengths.

As we will discuss in more detail below, at least for the system used in our study, the double-layer potentials, as determined from SFG, are in excellent agreement with those from an analysis of disjoining pressure isotherms of the same system but in a foam film (Figure 4).

We now want to discuss our results from the thin-film pressure balance, which are used to obtain $\chi_{S}^{(3)}$ (eq 10), as well as for direct verification of the potentials calculated by SFG (eq 9). To determine $\phi_{0}$ from disjoining pressure isotherms $\Pi(d)$ of foam films, the separation $d$ between the two charged surfaces needs to be determined (Figure 1c). $\Pi$ arises from the balance between electrostatic force, dispersion, and other intermolecular forces. ${ }^{13,18}$ In our case, we consider only the repulsive electrostatic $\Pi_{\mathrm{el}}$ and the attractive part $\Pi_{\mathrm{vdW}}$ of the van der Waals forces. ${ }^{9,10}$ Analysis of the experimentally determined disjoining pressure isotherms provides access to $\phi_{0}$. The generalized equation ${ }^{13,18}$ for $\Pi$ can be expressed for the case of monovalent (molecular) ions as follows

$$
\begin{aligned}
\Pi & =\Pi_{\mathrm{el}}+\Pi_{\mathrm{vdW}} \\
& \approx 64 k_{\mathrm{B}} T\left[\tanh \left(\frac{e \phi_{0}}{4 k_{\mathrm{B}} T}\right)\right]^{2} I \exp (-\kappa d)-\frac{A}{6 \pi d^{3}}
\end{aligned}
$$

Equation 12 can be now used to fit the experimental data $\Pi(d)$ with $\phi_{0}$ as the only free parameter (Supporting Information). For the Hamaker constant $A$, we used $3.7 \times 10^{-20} \mathrm{~J}$ for the air-water-air system. ${ }^{13}$ Note that the film thickness measured by optical methods is an equivalent thickness $h_{\text {eq }}$ that differs from the real physical thickness $d$ of the water core film, because the thickness of the adsorbate layer contributes to $h_{\mathrm{eq}}$. Although $d \approx h_{\text {eq }}$ is often assumed for simplicity, ${ }^{22}$ a more detailed analysis involves the exact value of $d=h_{\mathrm{eq}}-\Delta h_{\text {corr }}$, where $\Delta h_{\text {corr }}$ is a correction factor. ${ }^{18,50,71}$ We found a value of $3.2 \pm 0.6 \mathrm{~nm}$ for $\Delta h_{\text {corr }}$, wherein the standard deviation is due to approximations for the thickness and the refractive index of the adsorbate layer which are used in the data analysis (Supporting Information). Figure 3 shows a comparison

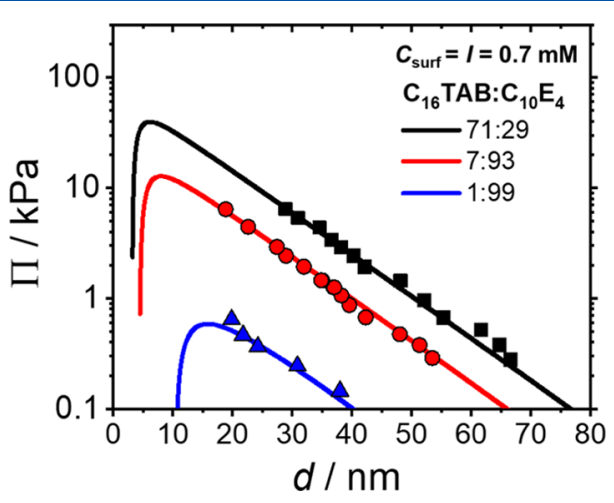

Figure 3. Disjoining pressure isotherms $\Pi(d)$ of foam films for $\left[\mathrm{C}_{16} \mathrm{TAB}\right] /\left[\mathrm{C}_{10} \mathrm{E}_{4}\right]$ mixing ratios with constant ionic strengths $I$ of 0.7 $\mathrm{mM}$ and a total concentration $C_{\text {surf }}$ of $0.7 \mathrm{mM}$. The mole percentage of each component of the mixtures was as indicated in the figure. Symbols: experimental data, solid lines: the best fit with eq 12 .

between theory and experiment for three selected surfactant $\left[\mathrm{C}_{16} \mathrm{TAB}\right] /\left[\mathrm{C}_{10} \mathrm{E}_{4}\right]$ mixing ratios. The results in Figure 3 demonstrate that decreasing the $\mathrm{C}_{16} \mathrm{TAB}$ concentration causes a progressive shift of the $\Pi(d)$ isotherms toward smaller film thicknesses $d$, which already points to a decrease in $\phi_{0}$, as one would also intuitively expect.

Figure 4 shows the results of our analysis from both methods (SFG and TFPB) for $\phi_{0, n}$ at the different mixing ratios, where $n$ refers to the molar fraction of $\mathrm{C}_{16} \mathrm{TAB}$. From a close comparison of Figure 4, we find an excellent agreement between the two complementary methods, which also brings strong support to our model and the above-mentioned assumptions for SFG analysis. Comparing our work with the recent report by Dreier et al., ${ }^{23}$ where SHG spectroscopy was combined with a vibrating plate capacitor method, our approach to combine SFG with a TFPB provides a better overlap between the latter techniques because they both depend strongly on the double-layer potential.

To test a possible bias of our procedure that could be caused by the initial choice of $\phi_{0, \text { TFPB }}$ in eq 10 , we calculated $\phi_{0, n}$ using different initial potentials $\phi_{0, \text { TFPB }}$ for calibration. From the latter, we calculated $\phi_{0, n}$ as explained above and present the mean values for the different initial conditions in Figure 4. This comparison is omitted for clarity in Figure 4 but is shown in Figure S4 of the Supporting Information. In addition, we demonstrate in Figure S5 of the Supporting Information that our analysis is also independent of the choice of the $\mathrm{O}-\mathrm{H}$ band. A separate analysis of the $\mathrm{O}-\mathrm{H}$ stretching bands at 3250 and $3450 \mathrm{~cm}^{-1}$ provides again an excellent agreement with the 


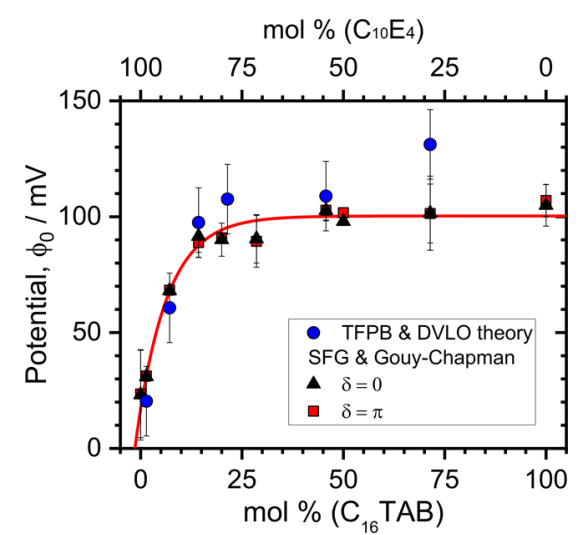

Figure 4. Comparison of $\phi_{0}$ determined by applying DLVO theory to fit the disjoining pressure isotherms, as obtained from a thin-film pressure balance (TFPB, circles in blue) with results from SFG spectroscopy taking into account the phase between $\chi_{\mathrm{S}}^{(2)}$ and $\chi_{\mathrm{S}}^{(3)}$ versus mole fraction of $\mathrm{C}_{16} \mathrm{TAB}$. All data points correspond to samples with the total surfactant concentration $\left[\mathrm{C}_{16} \mathrm{TAB}\right]+\left[\mathrm{C}_{10} \mathrm{E}_{4}\right]$ and the ionic strength fixed to $0.7 \mathrm{mM}$. The solid line guides the eye.

results from the TFPB. In addition, the choice of the phase between $\chi_{\mathrm{S}}^{(2)}$ and $\chi_{\mathrm{S}}^{(3)}$ being either 0 or $\pi$ does not change the results significantly (Figure 4). A close comparison of all data sets in Figures 4 and S4, S5 shows excellent agreement, which we take as further evidence for the robustness of our procedure, as well as for the feasibility of SFG spectroscopy for the determination of double-layer potentials at aqueous interfaces.

\section{SUMMARY AND CONCLUSIONS}

In summary, we demonstrated in this letter the ability of SFG spectroscopy to determine quantitatively double-layer potentials at liquid-gas interfaces by validating our results with complementary measurements of the disjoining pressure in foam films using a TFPB. For that, we have systematically changed $\phi_{0}$ by changing the bulk mixing ratio of nonionic $\mathrm{C}_{10} \mathrm{E}_{4}$ and cationic $\mathrm{C}_{16} \mathrm{TAB}$ surfactants, with the latter surfactant serving as the charge-determining species. Results from SFG spectroscopy on double-layer potentials are in excellent agreement with foam film results. The detailed analysis of the foam film data provided reliable $\phi_{0}$ values, which were used as initial inputs to obtain the a priori unknown values for third-order contributions to the electric susceptibility. We point out that compared with measurements with a TFPB, SFG can be seen as a fast and time-efficient method for addressing the charging state of fluid interfaces (1 day for all mixing ratios in SFG versus 1 day per mixing ratio for TFPB). Therefore, our new strategy, which uses the latest developments in the analysis of second-order optical spectroscopies, provides a reliable and validated method to measure $\phi_{0}$, which also accounts for the changes in the interfacial molecular structure. Furthermore, we expect that the procedure we describe can be applied to solid/liquid interfaces as well. ${ }^{31}$

\section{ASSOCIATED CONTENT}

\section{S Supporting Information}

The Supporting Information is available free of charge on the ACS Publications website at DOI: 10.1021/acs.jpcc.8b10097.

Sample preparation procedures and a table with the different mixing ratios used; $\mathrm{C}_{16} \mathrm{TAB}-$ and $\mathrm{C}_{10} \mathrm{E}_{4^{-}}$ measured surface tension isotherms; overview of the broadband SFG spectrometer; fitting procedures for SFG spectra and theoretical details; TFPB technique employed and the theoretical DLVO analysis of the film disjoining pressure isotherms (PDF)

\section{AUTHOR INFORMATION}

\section{Corresponding Author}

*E-mail: braunschweig@uni-muenster.de.

\section{ORCID $\odot$}

Natalia García Rey: 0000-0002-9175-3416

Björn Braunschweig: 0000-0002-6539-1693

\section{Notes}

The authors declare no competing financial interest.

\section{ACKNOWLEDGMENTS}

The authors are grateful for the funding from the European Research Council (ERC) under the European Union's Horizon 2020 research and innovation program (grant agreement no. 638278).

\section{REFERENCES}

(1) Adamson, A. W.; Gast, A. P. Physical Chemistry of Surfaces, 6the ed.; Wiley-Interscience Publication: New York, 1997.

(2) Bagchi, B. Water Dynamics in the Hydration Layer Around Proteins and Micelles. Chem. Rev. 2005, 105, 3197-3219.

(3) Jubb, A. M.; Hua, W.; Allen, H. C. Environmental Chemistry at Vapor/water Interfaces: Insights from Vibrational Sum Frequency Generation Spectroscopy. Annu. Rev. Phys. Chem. 2012, 63, 107-130.

(4) Johnson, C. M.; Baldelli, S. Vibrational Sum Frequency Spectroscopy Studies of the Influence of Solutes and Phospholipids at Vapor/Water Interfaces Relevant to Biological and Environmental Systems. Chem. Rev. 2014, 114, 8416-8446.

(5) Dalstein, L.; Potapova, E.; Tyrode, E. The Elusive Silica/Water Interface: Isolated Silanols Under Water as Revealed by Vibrational Sum Frequency Spectroscopy. Phys. Chem. Chem. Phys. 2017, 19, 10343-10349.

(6) Lützenkirchen, J.; Franks, G. V.; Plaschke, M.; Zimmermann, R.; Heberling, F.; Abdelmonem, A.; Darbha, G. K.; Schild, D.; Filby, A.; Eng, P.; et al. The Surface Chemistry of Sapphire-C: A Literature Review and a Study on Various Factors Influencing its IEP. Adv. Colloid Interface Sci. 2018, 251, 1-25.

(7) Schaefer, J.; Gonella, G.; Bonn, M.; Backus, E. H. G. SurfaceSpecific Vibrational Spectroscopy of the Water/Silica Interface: Screening and Interference. Phys. Chem. Chem. Phys. 2017, 19, $16875-16880$.

(8) Koepf, E.; Richert, M.; Braunschweig, B.; Schroeder, R.; Brezesinski, G.; Friess, W. Impact of Formulation $\mathrm{pH}$ on Physicochemical Protein Characteristics at the Liquid-Air Interface. Int. J. Pharm. 2018, 541, 234-245.

(9) Verwey, E. J. W.; Overbeek, J. T. G. Theory of the Stability of Lyophobic Colloids; Elsevier: Leiden, 1948.

(10) Derjaguin, B.; Landau, L. Theory of the Stability of Strongly Charged Lyophobic Sols and of the Adhesion of Strongly Charged Particles in Solutions of Electrolytes. Prog. Surf. Sci. 1993, 43, 30-59.

(11) Engelhardt, K.; Rumpel, A.; Walter, J.; Dombrowski, J.; Kulozik, U.; Braunschweig, B.; Peukert, W. Protein Adsorption at the Electrified Air-Water Interface: Implications on Foam Stability. Langmuir 2012, 28, 7780-7787.

(12) Braunschweig, B.; Schulze-Zachau, F.; Nagel, E.; Engelhardt, K.; Stoyanov, S.; Gochev, G.; Khristov, K.; Mileva, E.; Exerowa, D.; Miller, R.; et al. Specific Effects of $\mathrm{Ca}^{2+}$ Ions and Molecular Structure of $\beta$-Lactoglobulin Interfacial Layers that Drive Macroscopic Foam Stability. Soft Matter 2016, 12, 5995-6004.

(13) Israelachvili, J. N. Intermolecular and Surface Forces, 3rd ed.; Academic Press: Burlington, MA, 2011. 
(14) Hillier, A. C.; Kim, S.; Bard, A. J. Measurement of DoubleLayer Forces at the Electrode/Electrolyte Interface Using the Atomic Force Microscope: Potential and Anion Dependent Interactions. J. Phys. Chem. 1996, 100, 18808-18817.

(15) Liu, F.; Klaassen, A.; Zhao, C.; Mugele, F.; van den Ende, D. Electroviscous Dissipation in Aqueous Electrolyte Films with Overlapping Electric Double Layers. J. Phys. Chem. B 2018, 122, 933-946.

(16) Wu, B.; Liu, G.; Zhang, G.; Craig, V. S. J. Polyelectrolyte Multilayers under Compression: Concurrent Osmotic Stress and Colloidal Probe Atomic Force Microscopy. Soft Matter 2018, 14, 961-968.

(17) Stubenrauch, C.; von Klitzing, R. Disjoining Pressure in Thin Liquid Foam and Emulsion Films-New Concepts and Perspectives. J. Phys.: Condens. Matter 2003, 15, R1197-R1232.

(18) Platikanov, D.; Exerowa, D. Fundamentals of Foam Films. In Foam Films and Foams, Fundamentals and Applications; Exerowa, D. et al., Ed.; Progress in Colloid and Interface Science; CRC Press: Boca Raton, 2018; Chapter 3, Vol. 7.

(19) Hayes, P. L.; Chen, E. H.; Achtyl, J. L.; Geiger, F. M. An Optical Voltmeter for Studying Cetyltrimethylammonium Interacting with Fused Silica/Aqueous Interfaces at High Ionic Strength. J. Phys. Chem. A 2009, 113, 4269-4280.

(20) Wen, Y.-C.; Zha, S.; Liu, X.; Yang, S.; Guo, P.; Shi, G.; Fang, H.; Shen, Y. R.; Tian, C. Unveiling Microscopic Structures of Charged Water Interfaces by Surface-Specific Vibrational Spectroscopy. Phys. Rev. Lett. 2016, 116, No. 16101.

(21) Gonella, G.; Lütgebaucks, C.; de Beer, A. G. F.; Roke, S. Second Harmonic and Sum-Frequency Generation from Aqueous Interfaces Is Modulated by Interference. J. Phys. Chem. C 2016, 120, 9165-9173.

(22) Shahir, A. A.; Khristov, K.; Nguyen, K. T.; Nguyen, A. V.; Mileva, E. Combined Sum Frequency Generation and Thin Liquid Film Study of the Specific Effect of Monovalent Cations on the Interfacial Water Structure. Langmuir 2018, 34, 6844-6855.

(23) Dreier, L. B.; Bernhard, C.; Gonella, G.; Backus, E. H. G.; Bonn, M. Surface Potential of a Planar Charged Lipid-Water Interface. What Do Vibrating Plate Methods, Second Harmonic and Sum Frequency Measure? J. Phys. Chem. Lett. 2018, 5685-5691.

(24) Zhao, X.; Ong, S.; Eisenthal, K. B. Polarization of Water Molecules at a Charged Interface. Second Harmonic Studies of Charged Monolayers At the Air/Water Interface. Chem. Phys. Lett. 1993, 202, 513-520.

(25) Zhao, X.; Ong, S.; Wang, H.; Eisenthal, K. B. New Method for Determination of Surface $\mathrm{pKa}$ Using Second Harmonic Generation. Chem. Phys. Lett. 1993, 214, 203-207.

(26) Geiger, F. M. Second Harmonic Generation, Sum Frequency Generation, and Chi(3): Dissecting Environmental Interfaces with a Nonlinear Optical Swiss Army Knife. Annu. Rev. Phys. Chem. 2009, 60, 61-83.

(27) Ohno, P. E.; Saslow, S. A.; Wang, H.-F.; Geiger, F. M.; Eisenthal, K. B. Phase-Referenced Nonlinear Spectroscopy of the $\alpha$ Quartz/Water Interface. Nat. Commun. 2016, 7, No. 13587.

(28) Nihonyanagi, S.; Yamaguchi, S.; Tahara, T. Direct Evidence for Orientational Flip-Flop of Water Molecules at Charged Interfaces: a Heterodyne-Detected Vibrational Sum Frequency Generation Study. J. Chem. Phys. 2009, 130, No. 204704.

(29) Nihonyanagi, S.; Mondal, J. A.; Yamaguchi, S.; Tahara, T. Structure and Dynamics of Interfacial Water Studied by HeterodyneDetected Vibrational Sum-Frequency Generation. Annu. Rev. Phys. Chem. 2013, 64, 579-603.

(30) Ohno, P. E.; Wang, H.-F.; Geiger, F. M. Second-Order Spectral Lineshapes from Charged Interfaces. Nat. Commun. 2017, 8, No. 1032

(31) Ohno, P. E.; Wang, H.-F.; Paesani, F.; Skinner, J. L.; Geiger, F. M. Second-Order Vibrational Lineshapes from the Air/Water Interface. J. Phys. Chem. A 2018, 122, 4457-4464.

(32) Saint-Jalmes, A. Surfactant-Stabilized Foams. In Foam Films and Foams, Fundamentals and Applications; Exerowa, D. et al., Ed.;
Progress in Colloid and Interface Science; CRC Press: Boca Raton, 2018; Chapter 13, Vol. 7.

(33) Golemanov, K.; Denkov, N. D.; Tcholakova, S.; Vethamuthu, M.; Lips, A. Surfactant Mixtures for Control of Bubble Surface Mobility in Foam Studies. Langmuir 2008, 24, 9956-9961.

(34) Stubenrauch, C.; Claesson, P. M.; Rutland, M.; Manev, E.; Johansson, I.; Pedersen, J. S.; Langevin, D.; Blunk, D.; Bain, C. D. Mixtures of N-Dodecyl-Beta-D-Maltoside and Hexaoxyethylene Dodecyl Ether-Surface Properties, Bulk Properties, Foam Films, and Foams. Adv. Colloid Interface Sci. 2010, 155, 5-18.

(35) Carey, E.; Stubenrauch, C. A Disjoining Pressure Study of Foam Films Stabilized by Mixtures of a Nonionic $\left(\mathrm{C}_{12} \mathrm{DMPO}\right)$ and an Ionic Surfactant ( $\left.\mathrm{C}_{12} \mathrm{TAB}\right)$. J. Colloid Interface Sci. 2010, 343, 314323.

(36) Carey, E.; Stubenrauch, C. Free Drainage of Aqueous Foams Stabilized by Mixtures of a nonionic $\left(\mathrm{C}_{12} \mathrm{DMPO}\right)$ and an Ionic ( $\left.\mathrm{C}_{12} \mathrm{TAB}\right)$ Surfactant. Colloids Surf., A 2013, 419, 7-14.

(37) Jungwirth, P.; Finlayson-Pitts, B. J.; Tobias, D. J. Introduction: Structure and Chemistry at Aqueous Interfaces. Chem. Rev. 2006, 106, $1137-1139$.

(38) Zhang, Z.; Fenter, P.; Cheng, L.; Sturchio, N. C.; Bedzyk, M. J.; Předota, M.; Bandura, A.; Kubicki, J. D.; Lvov, S. N.; Cummings, P. T.; et al. Ion Adsorption at the Rutile-Water Interface: Linking Molecular and Macroscopic Properties. Langmuir 2004, 20, 49544969.

(39) Park, C.; Fenter, P. A.; Sturchio, N. C.; Nagy, K. L. Thermodynamics, Interfacial Structure, and $\mathrm{pH}$ Hysteresis of $\mathrm{Rb}^{+}$ and $\mathrm{Sr}^{2+}$ Adsorption at the Muscovite (001)-Solution Interface. Langmuir 2008, 24, 13993-14004.

(40) Liu, Y.; Yan, E. C. Y.; Zhao, X.; Eisenthal, K. B. Surface Potential of Charged Liposomes Determined by Second Harmonic Generation. Langmuir 2001, 17, 2063-2066.

(41) Melnichenko, Y. B. Small-Angle Scattering from Confined and Interfacial Fluids: Applications to Energy Storage and Environmental Science, 1st ed.; Springer: Cham, 2016.

(42) Jubb, A. M.; Hua, W.; Allen, H. C. Organization of Water and Atmospherically Relevant Ions and Solutes: Vibrational Sum Frequency Spectroscopy at the Vapor/Liquid and Liquid/Solid Interfaces. Acc. Chem. Res. 2012, 45, 110-119.

(43) de Beer, A. G. F.; Campen, R. K.; Roke, S. Separating Surface Structure and Surface Charge with Second-Harmonic and SumFrequency Scattering. Phys. Rev. B 2010, 82, No. 235431.

(44) Brown, M. A.; Abbas, Z.; Kleibert, A.; Green, R. G.; Goel, A.; May, S.; Squires, T. M. Determination of Surface Potential and Electrical Double-Layer Structure at the Aqueous ElectrolyteNanoparticle Interface. Phys. Rev. X 2016, 6, No. 011007.

(45) Fainerman, V. B.; Miller, R. Simple Method to Estimate Surface Tension of Mixed Surfactant Solutions. J. Phys. Chem. B 2001, 105, 11432-11438.

(46) Fainerman, V. B.; Vollhardt, D.; Emrich, G. Dynamics and Phase Transition in Adsorbed Monolayers of Sodium Dodecyl Sulfate/Dodecanol Mixtures. J. Phys. Chem. B 2001, 105, 4324-4330.

(47) Bergeron, V. Disjoining Pressures and Film Stability of Alkyltrimethylammonium Bromide Foam Films. Langmuir 1997, 13, 3474-3482.

(48) Santini, E.; Ravera, F.; Ferrari, M.; Stubenrauch, C.; Makievski, A.; Krägel, J. A Surface Rheological Study of nonionic Surfactants at the Water-Air Interface and the Stability of the Corresponding Thin Foam Films. Colloids Surf., A 2007, 298, 12-21.

(49) Lu, J. R.; Thomas, R. K.; Penfold, J. Surfactant Layers at the Air/Water Interface: Structure and Composition. Adv. Colloid Interface Sci. 2000, 84, 143-304.

(50) Kolarov, T.; Cohen, R.; Exerowa, D. Direct Measurement of Disjoining Pressure in Black Foam Films II. Films from Nonionic Surfactants. Colloids Surf. 1989, 42, 49-57.

(51) Shen, Y. R. Basic Theory of Surface Sum-Frequency Generation. J. Phys. Chem. C 2012, 116, 15505-15509.

(52) de Beer, A. G. F.; Samson, J.-S.; Hua, W.; Huang, Z.; Chen, X.; Allen, H. C.; Roke, S. Direct Comparison of Phase-Sensitive 
Vibrational Sum Frequency Generation with Maximum Entropy Method: Case Study of Water. J. Chem. Phys. 2011, 135, No. 224701.

(53) Gragson, D. E.; McCarty, B. M.; Richmond, G. L. Ordering of Interfacial Water Molecules at the Charged Air/Water Interface Observed by Vibrational Sum Frequency Generation. J. Am. Chem. Soc. 1997, 119, 6144-6152.

(54) Richmond, G. L. Molecular Bonding and Interactions at Aqueous Surfaces as Probed by Vibrational Sum Frequency Spectroscopy. Chem. Rev. 2002, 102, 2693-2724.

(55) Streubel, S.; Schulze-Zachau, F.; Weißenborn, E.; Braunschweig, B. Ion Pairing and Adsorption of Azo Dye/ $\mathrm{C}_{16} \mathrm{TAB}$ Surfactants at the Air-Water Interface. J. Phys. Chem. C 2017, 121, 27992-28000.

(56) Ong, S.; Zhao, X.; Eisenthal, K. B. Polarization of Water Molecules at a Charged Interface: Second Harmonic Studies of the Silica/Water Interface. Chem. Phys. Lett. 1992, 191, 327-335.

(57) Jena, K. C.; Covert, P. A.; Hore, D. K. The Effect of Salt on the Water Structure at a Charged Solid Surface: Differentiating Secondand Third-order Nonlinear Contributions. J. Phys. Chem. Lett. 2011, 2, $1056-1061$.

(58) Joutsuka, T.; Morita, A. Electrolyte and Temperature Effects on Third-Order Susceptibility in Sum-Frequency Generation Spectroscopy of Aqueous Salt Solutions. J. Phys. Chem. C 2018, 11407-11413.

(59) Pezzotti, S.; Galimberti, D. R.; Shen, Y. R.; Gaigeot, M.-P. Structural Definition of the BIL and DL: a New Universal Methodology to Rationalize Non-Linear $\chi^{(2)}(\omega)$ SFG Signals at Charged Interfaces, Including $\chi^{(3)}(\omega)$ Contributions. Phys. Chem. Chem. Phys. 2018, 20, 5190-5199.

(60) Tyrode, E.; Corkery, R. On the Charging of Carboxylic Acid Monolayers with Monovalent Ions at Low Ionic Strengths: Molecular Insight Revealed by Vibrational Sum Frequency Spectroscopy. J. Phys. Chem. C 2018, 28775-28786.

(61) Mysels, K. J.; Jones, M. N. Direct Measurement of the Variation of Double-layer Repulsion with Distance. Discuss. Faraday Soc. 1966, $42,42$.

(62) Kuntz, M. A New Implementation of the Humlicek Algorithm for the Calculation of the Voigt Profile Function. J. Quant. Spectrosc. Radiat. Transfer 1997, 57, 819-824.

(63) Ni, Y.; Skinner, J. L. Communication: Vibrational SumFrequency Spectrum of the Air-Water Interface, Revisited. J. Chem. Phys. 2016, 145, No. 031103.

(64) Oh-e, M.; Lvovsky, A. I.; Wei, X.; Shen, Y. R. Sum-Frequency Generation (SFG) Vibrational Spectroscopy of Side Alkyl Chain Structures of Polyimide Surfaces. J. Chem. Phys. 2000, 113, 88278832.

(65) Bell, G. R.; Bain, C. D.; Ward, R. N. Sum-Frequency Vibrational Spectroscopy of Soluble Surfactants at the Air/Water Interface. Faraday Trans. 1996, 92, 515.

(66) Urashima, S.-H.; Myalitsin, A.; Nihonyanagi, S.; Tahara, T. The Topmost Water Structure at a Charged Silica/Aqueous Interface Revealed by Heterodyne-Detected Vibrational Sum Frequency Generation Spectroscopy. J. Phys. Chem. Lett. 2018, 9, 4109-4114.

(67) Nakahara, H.; Shibata, O.; Moroi, Y. Examination of Surface Adsorption of Cetyltrimethylammonium Bromide and Sodium Dodecyl Sulfate. J. Phys. Chem. B 2011, 115, 9077-9086.

(68) Para, G.; Jarek, E.; Warszyński, P.; Adamczyk, Z. Effect of electrolytes on surface tension of ionic surfactant solutions. Colloids Surf., A 2003, 222, 213-222.

(69) Kumar, B.; Tikariha, D.; Ghosh, K. K. Effects of Electrolytes on Micellar and Surface Properties of Some Monomeric Surfactants. J. Dispersion Sci. Technol. 2012, 33, 265-271.

(70) Buchavzov, N.; Stubenrauch, C. A Disjoining Pressure Study of Foam Films Stabilized by Mixtures of Nonionic and Ionic Surfactants. Langmuir 2007, 23, 5315-5323.

(71) Duyvis, E. M. The Equilibrium Thickness of Free Liquid Films.

Ph.D. Thesis, Utrecht University: Utrecht, 1962. 